\title{
CRESCIMENTO DA CANDEIA PELA ADUBAÇÃO MINERAL E ORGÂNICA EM REJEITO DA MINERAÇÃO DE QUARTZITO
}

\author{
Cristiany Silva Amaral ${ }^{1}$, Enilson de Barros Silva ${ }^{2}$, Israel Marinho Pereira ${ }^{3}$, Bárbara Olinda Nardis ${ }^{4}$, \\ Neubert Homem Gonçalves ${ }^{5}$, Wander Gladson Amaral ${ }^{6}$ \\ ${ }^{1}$ Eng $^{\mathrm{a}}$. Florestal, M.Sc., UFVJM, Diamantina, MG, Brasil - cristianyamaral@yahoo.com.br \\ ${ }^{2}$ Eng. Agrônomo, UFVJM, Diamantina, MG, Brasil - ebsilva @ufvjm.edu.br \\ ${ }^{3}$ Eng. Florestal, UFVJM, Diamantina, MG, Brasil - imarinhopereira@gmail.com \\ ${ }^{4}$ Acadênico de Agronomia, UFVJM, Diamantina, MG, Brasil - babi.olinda@gmail.com \\ ${ }^{5}$ Eng. Florestal, Mestrando em Ciência Florestal, UFVJM, Diamantina, MG, Brasil - neubert.ef@ hotmail.com \\ ${ }^{6}$ Eng. Florestal, M.Sc., UFVJM, Diamantina, MG, Brasil - wander.amaral@yahoo.com.br \\ Recebido para publicação: 10/06/2013 - Aceito para publicação: 27/03/2014
}

\begin{abstract}
Resumo
A mineração tem promovido um grande aumento na ocorrência de áreas degradadas advindas de atividades mineradoras no Brasil. O objetivo deste trabalho foi avaliar o crescimento da candeia sob a influência da adubação mineral e orgânica quando cultivada em rejeito da mineração de quartzito. Conduziu-se um experimento em casa de vegetação em delineamento experimental inteiramente casualizado com quatro repetições. Os tratamentos consistiram de cinco combinações de adubação orgânica (AO) e mineral (AM) e um tratamento adicional do rejeito sem AO e AM (Controle). As combinações foram: $0 \% \mathrm{AO} / 100 \% \mathrm{AM} ; 25 \% \mathrm{AO} / 75 \% \mathrm{AM} ; 50 \% \mathrm{AO} / 50 \% \mathrm{AM} ; 75 \% \mathrm{AO} / 25 \% \mathrm{AM}$; $100 \% \mathrm{AO} / 0 \% \mathrm{AM}$. As doses de $100 \%$ de $\mathrm{AM}$ e $\mathrm{AO}$ foram de $0,15 \mathrm{~g}$ de $\mathrm{N}, 0,32 \mathrm{~g}$ de $\mathrm{P}_{2} \mathrm{O}_{5}, 0,18 \mathrm{~g}$ de $\mathrm{K}_{2} \mathrm{O}$ e $5 \mathrm{~g}$ de esterco de curral por $\mathrm{dm}^{3}$ de rejeito. A candeia respondeu positivamente à adubação mineral e orgânica com as doses recomendadas de $0,075 \mathrm{~g} \mathrm{~N}, 0,16 \mathrm{~g} \mathrm{P}_{2} \mathrm{O}_{5}$ e $0,09 \mathrm{~g} \mathrm{~K}_{2} \mathrm{O}$ e $2,5 \mathrm{~g}$ de esterco de curral, por $\mathrm{dm}^{3}$ de rejeito da mineração de quartzito. $\mathrm{O}$ acúmulo de nutrientes na espécie candeia foi na seguinte ordem na parte aérea: $\mathrm{K}>\mathrm{N}>\mathrm{P}>\mathrm{Ca}>\mathrm{Mg}>\mathrm{S}>\mathrm{Mn}>\mathrm{Fe}>\mathrm{B}>\mathrm{Zn}>\mathrm{Cu}$. Palavras-chave: Área degradada; teor adequado; estado nutricional; espécie nativa.
\end{abstract}

\begin{abstract}
Growth of Eremanthus erythropappus by mineral and organic fertilization on mining reject of quartzite. There is a large increase of degraded areas as result of mining activities in Brazil. This research aims to evaluate growth of the Eremanthus erythropappus on mining reject of quartzite, under the influence of mineral and organic fertilizer. We developed an experiment in a greenhouse, in completely randomized design with four replications. The treatments consisted of five combinations of mineral (MF) and organic $(\mathrm{OF})$ fertilizer, and an additional treatment of mining reject without MF and $\mathrm{OF}$ (witness). The combinations were as follows: $0 \% \mathrm{OF} / 100 \% \mathrm{MF} ; 25 \% \mathrm{OF} / 75 \% \mathrm{MF} ; 50 \% \mathrm{OF} / 50 \% \mathrm{MF} ; 75 \% \mathrm{OF} / 25 \% \mathrm{MF}$; $100 \% \mathrm{OF} / 0 \% \mathrm{MF}$. Doses of $100 \% \mathrm{MF}$ and OF were: $0,15 \mathrm{~g}$ of $\mathrm{N}, 0,32 \mathrm{~g}$ of $\mathrm{P}_{2} \mathrm{O}_{5}, 0,18 \mathrm{~g}$ of $\mathrm{K}_{2} \mathrm{O}$, and $5 \mathrm{~g}$ of bovine manure per $\mathrm{dm}^{3}$ of mining rejects. The Eremanthus erythropappus responded to mineral fertilization with recommended doses of $0,075 \mathrm{~g} \mathrm{~N}, 0,16 \mathrm{~g} \mathrm{P}_{2} \mathrm{O}_{5}$ and $0,09 \mathrm{~g} \mathrm{~K}_{2} \mathrm{O}$ and 2,5 $\mathrm{g}$ of bovine manure per $\mathrm{dm}^{3}$ of mining reject of quartzite. The accumulation of nutrients in the species Eremanthus erythropappus on the shoot was as follows: $\mathrm{K}>\mathrm{N}>\mathrm{P}>\mathrm{Ca}>\mathrm{Mg}>\mathrm{S}>\mathrm{Mn}>\mathrm{Fe}>\mathrm{B}>\mathrm{Zn}>\mathrm{Cu}$. Keywords: Degraded area; nutritional status; nutrients accumulation; native species.
\end{abstract}

\section{INTRODUÇÃO}

A ocorrência de áreas degradadas advindas da atividade de mineração no Brasil torna-se cada vez mais frequente e preocupante. Na região de Diamantina (MG), o declínio da mineração de diamante devido à interrupção das descobertas e o gradativo esgotamento das minas em operação, levou à descoberta e à exploração de maciços de quartzito, que proporciona a degradação da vegetação, da composição e de sua estrutura, tornando ambiente inóspito ao crescimento de plantas (WILLIAMSON et al., 2011). 
A atividade de mineração contribui para a redução dos processos de regeneração natural (CULLEN et al., 1998) e desfavorece o restabelecimento natural das espécies, pelo fato de haver a remoção da serapilheira, que contém sementes de plantas herbáceas, arbustivas e arbóreas que formam o banco de sementes e de plântulas que são partes integrantes da população vegetal (WILLIAMS, 1984). Assim, para que os processos de colonização de comunidades vegetais sejam acelerados, faz-se necessário plantios de enriquecimento nessas áreas degradadas, onde é crescente o desequilíbrio ecológico.

Das espécies autóctones que podem ser indicadas para compor os plantios nessas áreas degradadas, destaca-se a candeia (Eremanthus erythropappus (DC.) McLeish) (SCOLFORO et al., 2011; FEITOSA et al., 2008; SILVA et al., 2007). Essa espécie arbórea, pertencente à família Asteraceae, é indicada para a recuperação de áreas degradadas, pois ocorre naturalmente em solos pobres, arenosos e pedregosos (RIZZINI, 1979). Além disso, possui um sistema radicular profundo, com maior quantidade de raízes finas para melhor nutrir a planta, promovendo maior capacidade de crescimento e desenvolvimento. É considerada uma árvore de habitat especial, pois em áreas nativas é encontrada em altitudes em torno de $1.000 \mathrm{~m}$, em solos pobres, como Cambissolo e Litossolo, com afloramento de rochas (LORENZI, 1992).

Dessa maneira, a candeia apresenta atributos que justificam seu uso em plantios com fins para a restauração ambiental, mas o pouco conhecimento do comportamento dessa espécie, principalmente em relação às suas exigências nutricionais em áreas degradadas pela mineração de quartzito, torna sua utilização comprometida em programas de restauração.

O objetivo desse trabalho foi avaliar o crescimento de Eremanthus erythropappus sob a influência da adubação mineral e orgânica quando cultivada em rejeito da mineração de quartzito.

\section{MATERIAL E MÉTODOS}

O experimento foi conduzido em condições de casa de vegetação no Campus JK da Universidade Federal dos Vales do Jequitinhonha e Mucuri (UFVJM), em Diamantina, MG, Brasil

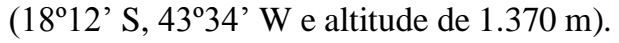

Para instalação do experimento, utilizou-se o rejeito da mineração de quartzito do município de Diamantina (MG). A amostra do substrato foi secada ao ar e passada em peneira de $2,0 \mathrm{~mm}$, sendo posteriormente realizada as análises química e de textura do substrato, conforme metodologias descritas em Silva (2009) e Empresa Brasileira de Pesquisa Agropecuária (EMBRAPA) (1997), respectivamente. Os resultados da análise química foram ajustados para o todo da constituição granulométrica do rejeito de quartzito (Tabela 1).

Tabela 1. Análise química e de textura do rejeito da mineração de quartzito.

Table 1. Chemical and texture analysis of the mining reject of quartzite.

\begin{tabular}{|c|c|c|c|c|c|c|c|c|c|c|c|c|c|}
\hline \multirow{2}{*}{ pH água } & $\mathbf{P}$ & $\mathbf{K}$ & $\mathbf{C a}$ & Mg & Al & $\mathbf{T}$ & $\mathbf{m}$ & $\mathbf{V}$ & MO & $\begin{array}{c}\text { Cascalho/ } \\
\text { calhaus }\end{array}$ & Areia & Silte & Argila \\
\hline & \multicolumn{2}{|c|}{ - mg.dm ${ }^{-3}$ - } & \multicolumn{4}{|c|}{--- cmol $_{\mathrm{c} .} \mathrm{dm}^{-3}$} & \multicolumn{2}{|c|}{--- \% ---- } & \multicolumn{5}{|c|}{ 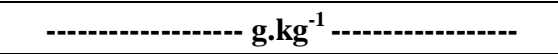 } \\
\hline 5,0 & 1,2 & 5,3 & 0,5 & 0,2 & 0,2 & 1,7 & 24 & 41 & 7,1 & 340,4 & 554,0 & 99,0 & 6,6 \\
\hline
\end{tabular}

$\mathrm{pH}$ água: relação solo-água 1:2,5. P e K: extrator Mehlich ${ }^{-1}$. Ca, $\mathrm{Mg}$ e Al: extrator $\mathrm{KCl} 1 \mathrm{~mol} \mathrm{~L}^{-1}$. T: capacidade de troca de cátions a pH 7,0. m: saturação de alumínio. V: saturação por bases. MO: matéria orgânica determinada através da multiplicação do resultado do carbono orgânico pelo método Walkey-Black por 1,724. Cascalho/calhaus: tamisação em peneira de 2,0 mm. Areia, silte e argila: método da pipeta.

O delineamento experimental utilizado foi inteiramente casualizado, com cinco combinações de adubação mineral (AM) e orgânica (AO) e um tratamento adicional (Controle), com quatro repetições cada. As quantidades de N, P e K e esterco de curral nas combinações de AM e AO e no controle são apresentados na tabela 2. As doses que corresponderam a $100 \%$ AM do rejeito se basearam em trabalho de Venturin et al. (2005) para candeia. A dose no trabalho de Venturin et al. (2005) de $0,46 \mathrm{~g}$ de $\mathrm{P}_{2} \mathrm{O}_{5}$ por $\mathrm{dm}^{3}$ para Latossolo Vermelho-Amarelo foi reduzida para $0,32 \mathrm{~g} \mathrm{de} \mathrm{P}_{2} \mathrm{O}_{5}$ por $\mathrm{dm}^{3}$ de rejeito, por ser substrato de textura muito arenosa (Tabela 1). N, P e K foram aplicados na forma de sulfato de amônio, superfosfato simples e cloreto de potássio, respectivamente. A dose de $5 \mathrm{~g}$ de esterco de curral por $\mathrm{dm}^{3} \mathrm{de}$ 
rejeito foi a recomendada pela Comissão de Fertilidade do Solo do Estado de Minas Gerais (CFSEMG) (1999). As análises químicas do esterco de curral ( $\mathrm{pH}=6,8 ; \mathrm{N}=6,0 ; \mathrm{P}=1,5 ; \mathrm{K}=1,3 ; \mathrm{Ca}=0,3 ; \mathrm{Mg}=$ 0,$4 ; \mathrm{S}=0,8 ; \mathrm{B}=0,18 ; \mathrm{Cu}=0,008 ; \mathrm{Fe}=27,2 ; \mathrm{Mn}=0,13$ e $\mathrm{Zn}=0,065 \mathrm{~g} \cdot \mathrm{kg}^{-1} ; \mathrm{C}$-orgânico $=178 \mathrm{~g}^{\mathrm{kg}} \mathrm{kg}^{-1} ; \mathrm{e}$ densidade $=0,69 \mathrm{~kg} \mathrm{dm}^{-3}$ ) foram realizadas segundo metodologia descrita por Melo e Silva (2008). A parcela experimental foi composta por vaso de plástico de $5 \mathrm{dm}^{3}$ sem furos com $3 \mathrm{dm}^{3}$ de rejeito de quartzito seco.

Tabela 2. Quantidades de $\mathrm{N}, \mathrm{P}_{2} \mathrm{O}_{5}$ e $\mathrm{K}_{2} \mathrm{O}$ e, esterco de curral para combinações de adubação mineral (AM) e orgânica $(\mathrm{AO})$ e do controle.

Table 2. Quantity of $\mathrm{N}, \mathrm{P}_{2} \mathrm{O}_{5}$ and $\mathrm{K}_{2} \mathrm{O}$ and bovine manure for combinations of mineral (MF) and organic (OF) fertilizer and control.

\begin{tabular}{|c|c|c|c|c|c|}
\hline AM & AO & $\mathbf{N}$ & $\mathbf{P}_{2} \mathbf{O}_{5}$ & $\begin{array}{l}\mathrm{K}_{2} \mathrm{O} \\
-\mathrm{g.dm}^{-3}\end{array}$ & Esterco de curral \\
\hline 100 & 0 & 0,150 & 0,32 & 0,180 & 0 \\
\hline 75 & 25 & 0,113 & 0,24 & 0,135 & 1,25 \\
\hline 50 & 50 & 0,075 & 0,16 & 0,090 & 2,50 \\
\hline 25 & 75 & 0,038 & 0,08 & 0,045 & 3,75 \\
\hline 0 & 100 & 0 & 0 & 0 & 5,00 \\
\hline Controle & & 0 & 0 & 0 & 0 \\
\hline
\end{tabular}

$\mathrm{Na}$ instalação do experimento, foram aplicados $0,119 \mathrm{~g}$ de calcário dolomítico por $\mathrm{dm}^{3}$ de rejeito para fornecimento de Ca $\left(0,05 \mathrm{~g} \cdot \mathrm{dm}^{-3}\right)$ e $\mathrm{Mg}\left(0,015 \mathrm{~g} \cdot \mathrm{dm}^{-3}\right)$. Na mesma época, foi realizada a adubação fosfatada e orgânica, de acordo com combinações de AM e AO. Uma semana antes do plantio das mudas, foi feita a adubação básica de micronutrientes: B (ácido bórico), $\mathrm{Cu}$ (cloreto de cobre), $\mathrm{Mn}$ (cloreto manganoso), Fe (cloreto ferroso) e $\mathrm{Zn}$ (sulfato de zinco) na dose de 0,0005 g de B, 0,0015 g de Cu, 0,02 g de $\mathrm{Mn}, 0,025 \mathrm{~g}$ de Fe e $0,001 \mathrm{~g}$ de $\mathrm{Zn}$ por $\mathrm{dm}^{3}$ de rejeito (VENTURIN et al., 2005).

As sementes de candeia foram coletadas em árvores no campo no município de Diamantina (MG) $\left(18^{\circ} 12^{\prime} \mathrm{S}, 43^{\circ} 34^{\prime} \mathrm{W}\right.$ e altitude de $\left.1.370 \mathrm{~m}\right)$. A semeadura foi realizada em canteiros de substrato composto por uma mistura nas mesmas proporções de solo esterilizado, casca de arroz carbonizada e areia. Foram transplantadas duas mudas em raiz nua com quatro pares de folhas por vaso. Após 30 dias, foi feito o desbaste, deixando-se apenas uma planta por vaso, com altura média de $0,11 \mathrm{~m}$ e diâmetro médio de $3,81 \mathrm{~mm}$. As adubações de $\mathrm{N}$ e $\mathrm{K}$ em cobertura foram parceladas em quatro vezes a cada 15 dias após o desbaste das mudas.

Após 120 dias do desbaste, realizou-se a avaliação da altura das mudas, diâmetro do caule medido na altura do colo e peso de massa seca da parte aérea (MSPA) e de raízes (MSR). O teor de nutrientes na parte aérea da candeia foi determinado segundo metodologia descrita por Malavolta et al. (1997). Para o cálculo do acúmulo de nutrientes da parte aérea da candeia, multiplicou-se o teor do nutriente pela massa seca.

Para se obter a porcentagem de AM e AO que proporcionou o melhor crescimento das mudas de candeia, os dados de altura e diâmetro de mudas, MSPA, MSR e relação MSR/MSPA foram submetidos a análise de variância multivariada, sendo que os tratamentos foram testados pelo critério de Wilks a 5\% (MORRISON, 1967). Constatada a significâncias para o efeito dos tratamentos, adotou-se o processo das variáveis canônicas (FERREIRA; DUARTE, 1992), obtendo-se, assim, a variável canônica com maior porcentagem da variação total (autovalor), a partir da qual foram calculados os escores para cada unidade experimental, submetidos em seguida à análise de variância univariada convencional. Modelos de regressão foram ajustados para as médias dos escores das combinações de AM e AO como variáveis dependentes das porcentagens de AM. A média dos escores do controle foi comparada com a dos demais tratamentos pelo teste de $\mathrm{F}$ a $5 \%$ de significância.

Os dados de teor e acumulado de nutrientes na parte aérea da candeia foram submetidos a análise de variância. Ajustaram-se equações de regressão para retratar as variações do teor e acúmulo de nutrientes na parte aérea, sendo a variável dependente, em função das variações da porcentagem de AM, variável independente. A média do controle foi comparada com a média das porcentagens de AM pelo teste de $\mathrm{F}$ a $5 \%$. Pela substituição da porcentagem de AM que proporcionou o máximo crescimento da 
candeia nessas equações de regressão, obtiveram-se o teor adequado e acumulado de nutrientes na parte aérea da candeia. Foi utilizado o programa SAS for Windows nos procedimentos estatísticos.

\section{RESULTADOS E DISCUSSÃO}

Pela análise de variância multivariada, a primeira Variável Canônica (VC) totalizou, isoladamente, $91 \%$ da variação total, o que é um valor mais do que satisfatório nos estudos realizados com essa técnica (FERREIRA; DUARTE, 1992). Por essa razão, seus escores foram escolhidos para a definição da primeira VC, representando o crescimento das mudas de candeia (VC $=0,3230 *$ Diâmetro 3,0241*Altura - 0,2274*MSPA + 0,8934*MSR - 1,0723*Relação MSR/MSPA). Verificou-se que o máximo crescimento foi atingido com $50 \%$ de $\mathrm{AM}$ e $50 \%$ de $\mathrm{AO}$ (Figura 1), que corresponde à dose de $0,15 \mathrm{~g}$ de $\mathrm{N}, 0,32 \mathrm{~g}$ de $\mathrm{P}_{2} \mathrm{O}_{5}, 0,18 \mathrm{~g}$ de $\mathrm{K}_{2} \mathrm{O}$ e $5 \mathrm{~g}$ de esterco de curral por $\mathrm{dm}^{3}$ de rejeito de quartzito. Esse efeito no crescimento pode ser estar relacionado às modificações nas propriedades físicas do substrato, que, por sua vez, influenciam na capacidade de infiltração e retenção de água, drenagem, aeração e penetração do sistema radicular (VARANINE et al., 1993).

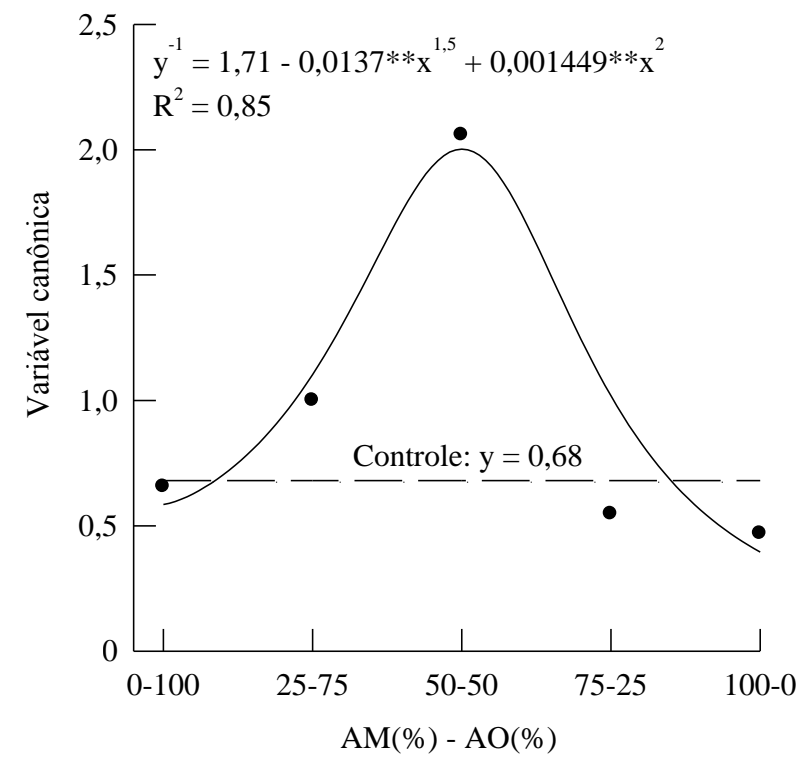

Figura 1. Escore da variável canônica da análise multivariada em função da porcentagem de Adubação mineral (AM) e ORGÂNICA (AO) aplicada em rejeito da mineração de quartzito no crescimento de mudas de candeia (** significativo a $1 \%$ pelo teste de $t$ ).

Figure 1. Canonic variable score of multivariate analysis as a function of the Percentage of Mineral (MF) and Organic $(\mathrm{OF})$ fertilization on mining reject of quartzite in the growth of seedlings of Eremanthus erythropappus (** significant to $1 \%$ by t test).

O crescimento das plantas de candeia foi maior na combinação de 50\% AM e 50\% AO (Figura 1), mostrando que a AM e AO são fundamentais no crescimento inicial da espécie em rejeito de quartzito. Resposta positiva a AM e AO foram encontradas em erva-mate (Ilex paraguariensis St.-Hil.) cultivada em Latossolo Vermelho com aplicação de 80,5 g N, $25 \mathrm{~g} \mathrm{P}_{2} \mathrm{O}_{5}, 75 \mathrm{~g} \mathrm{~K}_{2} \mathrm{O}$ e 4,5 kg de cama de aviário por planta (PANDOLFO et al., 2003), e em três espécies florestais (Cedrela odorata L., Schinus terebinthifolius Raddi e Acacia mangium var. holosericea C. White) com aplicação de adubo orgânico nas doses de 7 g/cova de NPK-20-05-20 e 1 L/cova de esterco de galinha e bovino (SOUZA et al., 2006). A adubação orgânica resulta em efeitos benéficos na formação de mudas de espécies florestais, como os relatados para oiti (Licania tomentosa Benth.) adubado com composto de lixo urbano (ALVES; PASSONI, 1997) e angelim (Andira fraxinifolia Benth.) adubado com esterco bovino (CARVALHO FILHO et al., 2004). 
Respostas positivas somente com adubação mineral N, P e K foram obtidos em candeia (VENTURIN et al., 2005) e com outras espécies florestais, como candiúva (Trema micrantha L. Blumes) (VENTURIN et al., 2000), aroeirinha (Schinus terenbinthifolius), paineira (Chorisia speciosa St.-Hil.) e jambolão (Sygygium jambolanum Lam.) (FERNANDES et al., 2000), sete-cascas (Samanea inopinata (Harms) Ducke) (CRUZ et al., 2006) e grápia (Apuleia leiocarpa (Vog.) Macbride) em Argissolo Vermelho distrófico arênico (NICOLOSO et al., 2009).

Os teores de todos os nutrientes foram influenciados pela $\mathrm{AM}$ e $\mathrm{AO}$, que diferiram do controle, exceto $\mathrm{Fe}$ e $\mathrm{Cu}$ (Tabela 3). A avaliação do estado nutricional da candeia permitiu obter os teores adequados de nutrientes (Tabela 3) em área degradada pela mineração de quartzito. Não existem informações quanto ao teor foliar de nutrientes para a candeia nessas condições. Os teores foliares de nutrientes na parte aérea foram comparados com teores adequados para essências florestais (MALAVOLTA et al., 1997).

$\mathrm{O}$ teor adequado de $\mathrm{N}$ na parte aérea da candeia cultivada em rejeito de quartzito no maior crescimento das mudas de candeia (Tabela 3) está dentro da faixa adequada para essências florestais,

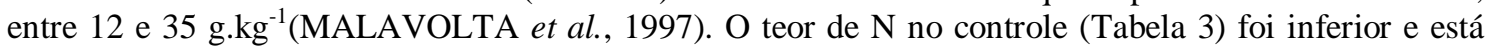
abaixo da faixa adequada (MALAVOLTA et al., 1997), confirmando a importância da AM e AO quanto à disponibilidade de $\mathrm{N}$ para demanda nutricional da espécie. No estádio juvenil, a demanda de $\mathrm{N}$ é muito elevada (GONÇALVES et al., 2000) e a maior absorção e assimilação de N é aceita como a principal causa da resposta em crescimento (VAZ et al., 2002).

Tabela 3. Equações de regressão ajustadas para os teores de nutrientes na parte aérea de candeia com variável dependente $(\hat{y})$ em função das variações da porcentagem da adubação mineral (x) aplicada em rejeito da mineração de quartzito, teor de nutrientes correspondente a $50 \%$ de adubação mineral (AM) e no controle, num período de 120 dias.

Table 3. Regression equations adjusted to concentrations of nutrients in the shoot of Eremanthus erythropappus with dependent variable ( $\hat{y})$ as a function of variations in the percentage of mineral fertilizer (x) applied on the mining reject of quartzite, nutrient concentration corresponding to $50 \%$ of mineral fertilizer (MF) and in control along 120 days.

\begin{tabular}{|c|c|c|c|c|}
\hline Nutriente $^{(\mathbf{1})}$ & Equação de regressão & $\mathbf{R}^{2}$ & $\mathbf{A M}^{(2)}$ & Controle $^{(3)}$ \\
\hline $\mathrm{N}$ & $\hat{y}=11,21+0,1696 * * x-0,0013 * * x^{2}$ & 0,83 & 16,44 & $9,05^{*}$ \\
\hline $\mathrm{P}$ & $\hat{\mathrm{y}}=0,85+0,0815 * * \mathrm{x}$ & 0,93 & 4,93 & $2,44 *$ \\
\hline $\mathrm{K}$ & $\hat{y}=15,13+0,1013 * * x$ & 0,88 & 20,20 & $9,65^{*}$ \\
\hline $\mathrm{Ca}$ & $\hat{\mathrm{y}}=2,49+0,0209 * * \mathrm{x}-0,0002 * * \mathrm{x}^{2}$ & 0,86 & 3,02 & $2,40^{*}$ \\
\hline $\mathrm{Mg}$ & $\hat{y}=0,52+0,0496^{* *} x-0,0005^{* *} x^{2}$ & 0,83 & 1,83 & $0,39 *$ \\
\hline $\mathrm{S}$ & $\hat{y}=2,79-0,0242 * * x$ & 0,91 & 1,35 & $0,70 *$ \\
\hline B & $\hat{y}=129,79-3,1977 * * x+0,0320 * * x^{2}$ & 0,85 & 49,85 & $44,03 *$ \\
\hline $\mathrm{Cu}$ & $\hat{y}=\bar{y}=14,50$ & - & $14,50^{(4)}$ & 10,76 \\
\hline $\mathrm{Fe}$ & $\hat{y}=\bar{y}=78,35$ & - & $78,35^{(4)}$ & 90,33 \\
\hline $\mathrm{Mn}$ & $\hat{y}=375,44+3,7650^{* * x}$ & 0,95 & 563,69 & $270,01 *$ \\
\hline $\mathrm{Zn}$ & $\hat{\mathrm{y}}=10,94+0,1948^{* *} \mathrm{x}$ & 0,97 & 20,69 & $12,50 *$ \\
\hline
\end{tabular}

**significativo a $1 \%$ pelo teste de $\mathrm{t} .{ }^{(1)}$ Macronutrientes em $\mathrm{g} \cdot \mathrm{kg}^{-1}$ e micronutrientes em $\mathrm{mg} \cdot \mathrm{kg}^{-1}$. ${ }^{(2)}$ Teores de nutrientes na aplicação de $50 \%$ AM e $50 \%$ AO. ${ }^{(3)} *$ Diferencia do controle dos demais tratamentos pelo teste F a $5 \%$. ${ }^{(4)}$ Teor médio das combinações de $\mathrm{AM}$ e AO sem ajuste de equação de regressão.

A concentração do $\mathrm{P}$ na parte aérea de candeia na combinação de $50 \%$ AM e $\mathrm{AO}$ e no controle (Tabela 3) encontra-se acima da faixa adequada de 1,0 a $2,3 \mathrm{~g} \mathrm{~kg}^{-1}$ para essências florestais (MALAVOLTA et al., 1997). Observa-se que até mesmo o controle apresentou concentração de P acima da faixa adequada para espécies florestais. Esse comportamento pode ser explicado pela textura arenosa do substrato e pela presença da matéria orgânica (Tabela 1). A disponibilidade de P no solo é um fator que interfere diretamente na sua absorção pelas plantas (MACHADO et al., 2011). Essa disponibilidade pode ser influenciada pela textura do solo, sendo que a adsorção de $\mathrm{P}$ em solos arenosos é menor que em solos argilosos (FALCÃO; SILVA, 2004) e que a adsorção de fosfato é reduzida na presença de matéria orgânica, que é também fonte de P para as plantas (RHEINHEIMER et al., 2000; CASSOL et al., 2001). 
Espécies arbóreas tendem a apresentar elevada capacidade de absorção de P na fase inicial de crescimento, podendo até apresentar significativo potencial de acúmulo de P-inorgânico no vacúolo das células (GRESPAN, 1997). P é nutriente considerado determinante no crescimento das plantas, uma vez que tem considerável importância no metabolismo de carbono e açúcares fosfatados (GRANT et al., 2001).

O teor foliar de K (Tabela 3) para a espécie está acima da faixa adequada (10 a $15{\mathrm{~g} . \mathrm{kg}^{-1}}^{-1}$ para essências florestais (MALAVOLTA et al., 1997). Na parte aérea do tratamento controle, verificou-se o menor teor de K (Tabela 3), possivelmente devido ao fato de não ter sido aplicado qualquer tipo de fertilizante, considerando que o teor de $\mathrm{K}$ na massa seca varia com a disponibilidade do nutriente no solo (KANO et al., 2010). No presente estudo, observou-se que $\mathrm{K}$ foi o elemento encontrado em maior concentração na parte aérea da candeia. Tal fato deveu-se, muito provavelmente, às características de fertilidade do substrato após a fertilização mineral e orgânica, uma vez que o esterco de curral é uma fonte orgânica de K para as plantas (GALVÃO et al., 2008).

$\mathrm{Na}$ massa seca da parte aérea de candeia cultivada em rejeito de quartzito com $50 \%$ de AM e $\mathrm{AO}$, os teores de $\mathrm{Ca}$ e de $\mathrm{Mg}$ (Tabela 3 ) estão dentro da faixa adequada para essências florestais, de 3 a 12 g.kg ${ }^{-1}$ de Ca e de 1,5 a 5,0 g.kg ${ }^{-1}$ de Mg (MALAVOLTA et al., 1997). Esses teores de Ca e de Mg do substrato foram supridos via aplicação de calcário dolomítico para fornecimento de $\mathrm{Ca}$ e $\mathrm{Mg}$, considerados suficientes para suprir as necessidades das plantas nessa fase.

Quanto ao teor de S no controle, este diferiu das combinações de AM e AO (Tabela 3). Enquanto que no maior crescimento da candeia o teor de $\mathrm{S}$ se encontra dentro da faixa adequada (Tabela 3), já no controle o teor encontra-se abaixo da faixa $\left(1,4\right.$ a 1,6 g. $\left.\mathrm{kg}^{-1}\right)$ (MALAVOLTA et al., 1997). Essa diferença observada, provavelmente, deve-se ao fato de que a quantidade de $\mathrm{S}$ no substrato na melhor combinação de AM e AO pode se dar pelo uso do sulfato de amônio como fonte de $\mathrm{N}$ e de superfosfato simples como fonte de $\mathrm{P}$, e, ainda, pela quantidade de matéria orgânica adicionada. A manutenção de teores adequados de matéria orgânica no solo é fundamental para o armazenamento de $\mathrm{S}$ orgânico para suprimento constante de $\mathrm{S}$ às plantas (OSÓRIO FILHO, 2006).

Em relação ao teor de micronutrientes no controle, tem-se que foram inferiores (Tabela 3) quando comparados aos teores na parte área da candeia no maior crescimento (50\% AM e AO). No entanto, todos os micros, exceto Mn, no controle tiveram teores dentro da faixa adequada preconizada por Malavolta et al. (1997) para essências nativas. Os teores de Mn foram relativamente altos considerando os teores adequados preconizados por Malavolta et al. (1997), de 15 a $60 \mathrm{mg} \cdot \mathrm{kg}^{-1}$, sendo que altas concentrações de Mn estão de acordo com o comportamento das essências florestais (REISSMANN et al., 1983).

Os valores encontrados para os teores dos micronutrientes $\mathrm{Cu}, \mathrm{Fe}, \mathrm{Zn}$ e $\mathrm{B}$ na parte aérea da candeia na melhor combinação de $\mathrm{AM}$ e $\mathrm{AO}$ estão dentro da faixa de variação considerada adequada, entre 10 e 70 para o $\mathrm{Cu}$, de 25 a 200 para o Fe, de 5 a 60 para o $\mathrm{Zn}$ e de 10 a $70 \mathrm{mg} \cdot \mathrm{kg}^{-1}$ para o B (MALAVOLTA et al., 1997). O teor adequado desses micronutrientes na parte aérea da candeia também explica o maior crescimento da espécie, apesar de suas baixas concentrações dentro dos tecidos e dos órgãos das plantas. Enquanto o teor de $\mathrm{Cu}$ atua em diversos processos fisiológicos, o $\mathrm{Zn}$ atua nos processos metabólicos das plantas. Já o B atua na dominância das gemas apicais e crescimento das plantas, e o Fe é constituinte de enzimas (KIRKBY; RÖMHELD, 2007).

Não existe informação detalhada quanto ao acúmulo de nutrientes por candeia em áreas degradadas pela extração de quartzito. Verificou-se neste trabalho efeito significativo $(\mathrm{P}<0,05)$ para acumulação de todos os nutrientes na parte aérea da candeia em função da porcentagem de $\mathrm{AM}$ e $\mathrm{AO}$ (Tabela 4).

Verifica-se que os valores acumulados de nutrientes pela espécie no controle foram sempre inferiores (Tabela 4) em relação ao de 50\% AM e AO. Isso se deve, de modo geral, ao menor crescimento das mudas no controle (Figura 1). Os valores acumulados para os macronutrientes na parte aérea apresentaram a seguinte ordem de acúmulo: $\mathrm{K}>\mathrm{N}>\mathrm{P}>\mathrm{Ca}>\mathrm{Mg}>\mathrm{S}$ (Tabela 4). Dentre os micronutrientes, na parte aérea a ordem decrescente das quantidades acumuladas ocorreu para $\mathrm{Mn}>\mathrm{Fe}>\mathrm{B}>\mathrm{Zn}>\mathrm{Cu}$ (Tabela 4). $\mathrm{O}$ acúmulo de macronutrientes em plantas de mogno foi diferente e na seguinte ordem com adubação completa: $\mathrm{N}>\mathrm{Ca}>\mathrm{K}>\mathrm{S}>\mathrm{Mg}>\mathrm{P}$ (WALLAU et al., 2008).

Para fins de utilização da espécie em projetos de restauração, tem-se que o acúmulo de nutrientes pela candeia foi inferior aos encontrados por Cunha et al. (1993) em Floresta Estacional Decidual. No 
entanto, esse mesmo autor obteve maior acúmulo de $\mathrm{K}$ seguido de $\mathrm{N}$, conforme o presente trabalho. Em Floresta Estacional Semidecidual Submontana, Oliveira (1997) encontrou produção de serapilheira de 14.715,97 kg.ha- ${ }^{-1}$.no ${ }^{-1}$, com retorno de macronutrientes na serapilheira na seguinte ordem: $\mathrm{Ca}>\mathrm{N}>\mathrm{K}>\mathrm{Mg}>\mathrm{P}$, não apresentando a mesma sequência de acúmulo para candeia.

Tabela 4. Equações de regressão ajustadas para o acúmulo de nutrientes na parte aérea de candeia com variável dependente ( $\hat{y})$ em função das variações da porcentagem da adubação mineral (x) aplicada em rejeito da mineração de quartzito, teor de nutrientes correspondente a $50 \%$ de adubação mineral (AM) e no controle, num período de 120 dias.

Table 4. Regression equations adjusted to accumulation of nutrients in the shoot of Eremanthus erythropappus with dependent variable ( $\hat{y})$ as a function of variations in the percentage of mineral fertilizer (x) applied on the mining reject of quartzite, nutrient concentration corresponding to $50 \%$ of mineral fertilizer (MF) and in control along 120 days.

\begin{tabular}{|c|c|c|c|c|}
\hline Nutriente $^{(1)}$ & Equação de regressão & $\mathbf{R}^{2}$ & $\mathbf{A M}^{(2)}$ & Controle $^{(3)}$ \\
\hline $\mathrm{N}$ & $\hat{\mathrm{y}}^{-1}=0,03-0,00032 * * \mathrm{x}^{1,5}+0,000036 * * \mathrm{x}^{2}$ & 0,99 & 136,35 & $13,76^{*}$ \\
\hline $\mathrm{P}$ & $\hat{y}^{-1}=0,30+0,00036^{* *} x^{1,5}-0,056^{* *} x^{0,5}$ & 0,93 & 31,44 & $3,71 *$ \\
\hline $\mathrm{K}$ & $\hat{\mathrm{y}}^{-1}=0,03-0,00095 * * \mathrm{x}+0,000011^{* *} \mathrm{x}^{2}$ & 0,98 & 153,84 & $14,68 *$ \\
\hline $\mathrm{Ca}$ & $\hat{y}^{-1}=0,16-0,00180 * * x^{1,5}+0,000203 * * x^{2}$ & 0,99 & 27,18 & $3,65^{*}$ \\
\hline $\mathrm{Mg}$ & $\hat{y}^{-1}=0,82-0,05538 * * x+0,005667 * * x^{1,5}$ & 0,99 & 18,77 & $0,59 *$ \\
\hline S & $\hat{y}^{-1}=0,13-0,00025 * * x^{2}+0,000033 * * x^{2,5}$ & 0,99 & 11,57 & $1,06^{*}$ \\
\hline B & $\hat{\mathrm{y}}^{-1}=0,002+0,00011 * * \mathrm{x}-0,000744 * * \mathrm{x}^{0,5}$ & 0,96 & 311,80 & $66,97 *$ \\
\hline $\mathrm{Cu}$ & $\hat{\mathrm{y}}^{-1}=0,02-0,00019 * * \mathrm{x}^{1,5}+0,000021 * * \mathrm{x}^{2}$ & 0,95 & 85,78 & $15,41 *$ \\
\hline $\mathrm{Fe}$ & $\hat{\mathrm{y}}^{-1}=0,01-0,00052 * * \mathrm{x}+0,000048 * * \mathrm{x}^{1,5}$ & 0,92 & 567,64 & $137,40 *$ \\
\hline $\mathrm{Mn}$ & $\hat{\mathrm{y}}^{-1}=0,001-0,000034 * * \mathrm{x}+0,00000037 * * \mathrm{x}^{2}$ & 0,96 & 4333,55 & $410,68 *$ \\
\hline $\mathrm{Zn}$ & $\hat{\mathrm{y}}^{-1}=0,04-0,00239 * * \mathrm{x}+0,000231^{* *} \mathrm{x}^{1,5}$ & 0,98 & 195,42 & $19,01 *$ \\
\hline
\end{tabular}

\section{CONCLUSÕES}

- Houve resposta positiva das mudas de Eremanthus erythropappus à adubação mineral e orgânica com as doses recomendadas de 0,075 $\mathrm{g} \mathrm{N}, 0,16 \mathrm{~g} \mathrm{P}_{2} \mathrm{O}_{5}, 0,09 \mathrm{~g} \mathrm{~K}_{2} \mathrm{O}$ e 2,5 g esterco de curral por $\mathrm{dm}^{3}$ de rejeito da mineração de quartzito.

- O acúmulo de nutrientes na parte aérea da candeia nas doses recomendadas foi na seguinte ordem: $\mathrm{K}>\mathrm{N}>\mathrm{P}>\mathrm{Ca}>\mathrm{Mg}>\mathrm{S}$ (para macro) e $\mathrm{Mn}>\mathrm{Fe}>\mathrm{B}>\mathrm{Zn}>\mathrm{Cu}$ (para micro).

\section{AGRADECIMENTOS}

Ao Conselho Nacional de Desenvolvimento Cientifico e Tecnológico, pela bolsa de mestrado. À Universidade Federal dos Vales Jequitinhonha e Mucuri, pela infraestrutura necessária para a condução do experimento.

\section{REFERÊNCIAS}

ALVES, W. L.; PASSONI, A. A. Composto e vermicomposto de lixo urbano na produção de mudas de oiti (Licania tomentosa Benth.) para arborização. Pesquisa Agropecuária Brasileira, Brasília, v. 32, n. 10, p. 1053 - 1058, 1997.

CARVAlHO FILHO, J. L. S.; ARRIGONI-BLANK, M. F.; BLANK, A. F. Produção de mudas de angelim (Andira fraxinifolia Benth.) em diferentes ambientes, recipientes e substratos. Revista Ciência Agronômica, Fortaleza, v. 35, n. 1, p. 61 - 67, 2004.

CASSOL, P. C.; GIANELlO, C.; COSTA, V. E. U. Frações de fósforo em estrumes e sua eficiência como adubo fosfatado. Revista Brasileira Ciência do Solo, Viçosa, v. 25, n. 6, p. 635 - 644, 2001. 
COMISSÃO DE FERTILIDADE DO SOLO DO ESTADO DE MINAS GERAIS (CFSEMG). Adubação orgânica. In: RIBEIRO, A. C.; GUIMARÃES, P. T. G.; ALVAREZ V., V. H. (Ed.). Recomendações para o uso de corretivos e fertilizantes em Minas Gerais. Viçosa: CFSEMG, 1999. p. 87 - 92.

CRUZ, C. A. F.; PAIVA, H. N.; GUERRERO, C. R. A. Efeito da adubação nitrogenada na produção de mudas de sete-cascas (Samanea inopinata (Harms) Ducke). Revista Árvore, Viçosa, v. 30, n. 4, p. 537 $546,1995$.

CULLEN, W. R.; WHEATER, C. P.; DUNLEAVY, P. J. Establishment of species-rich vegetation on reclaimed limestone quarry faces in Derbyshire, UK. Biological Conservation, Boston, v. 84, n. 1, p. 25 - 33, 1998.

CUNHA, G. C.; GRENDENE, L. A.; DURLO, M. A.; BRESSAN, D. A. Dinâmica nutricional em floresta estacional decidual com ênfase aos minerais provenientes da deposição da serapilheira. Ciência Florestal, Santa Maria, v. 3, n. 1, p. 35 - 64, 1993.

EMPRESA BRASILEIRA DE PESQUISA AGROPECUÁRIA (EMBRAPA). Centro Nacional de Pesquisa de Solos. Manual de métodos de análise de solo. Rio de Janeiro: CNPS, 1997. 212 p.

FALCÃO, N. P. S.; SILVA, J. R. A. Características de adsorção de fósforo em alguns solos da Amazônia Central. Acta Amazônica, Manaus, v. 34, n. 3, p. 337 - 342. 2004.

FEITOSA, S. S.; DAVIDE, A. C.; TONETTI, O. A. O.; FABRICANTE, J. R.; LUI, J. J. Estudos de viabilidade de sementes de candeia Eremanthus erythropappus (DC.). MacLeish, por meio de testes de germinação e raios X. Floresta, Curitiba, v. 39, n. 2, p. 393 - 399, 2008.

FERNANDES, L. A.; FURTINI NETO, A. F.; FONSECA, F. C.; VALE, F. R. Crescimento inicial, níveis críticos de fósforo e frações fosfatadas em espécies florestais. Pesquisa Agropecuária Brasileira, Brasília, v. 35, n. 6, p. 1191 - 1198, 2000.

FERREIRA, D. F.; DUARTE, G. S. Eficiência da análise de variância multivariada comparada à análise de variância univariada em experimentos com soja. Ciência e Prática, Lavras, v. 16, n. 2, p. 229 - 232, 1992.

GALVÃO, S. R. S.; SALCEDO, I. H.; OLIVEIRA, F. F. Acumulação de nutrientes em solos arenosos adubados com esterco bovino. Pesquisa Agropecuária Brasileira, Brasília, v. 43, n. 1, p. 99 - 105, 2008.

GONÇALVES, J. L. M.; STAPE, J. L.; BENEDETTI, V.; FESSEL, V. A. G.; GAVA, J. L. Reflexos do cultivo mínimo e intensivo do solo em sua fertilidade e na nutrição das árvores. In: GONÇALVES, J. L. M.; BENEDETTI, V. (Eds.). Nutrição e fertilização florestal. Piracicaba: Instituto de Pesquisas e Estudos Florestais, 2000. p. 3 - 57.

GRANT, C. A.; FLATEN, D. N.; TOMASIEWICZ, D. J.; SHEPPARD, S. C. A importância do fósforo no desenvolvimento inicial da planta. Informações Agronômicas, Piracicaba, n. 95, p. 1 - 5, 2001.

GRESPAN, S. L. Produção e eficiência nutricional de clones de eucalipto no norte do Espírito Santo e suas relações com características do solo. $81 \mathrm{f}$. Dissertação (Mestrado em Solos e Nutrição de Plantas) - Universidade Federal de Viçosa, Viçosa, 1997.

KANO, C.; CARDOSO, A.; VILLAS BÔAS, R. L. Influência de doses de potássio nos teores de macronutrientes em plantas e sementes de alface. Horticultura Brasileira, Campinas, v. 28, n. 3, p. 287 291, 2010.

KIRKBY, E. A.; RÖMHELD, V. Micronutrientes na fisiologia de plantas: Funções absorção e mobilidade. Informações Agronômicas, Piracicaba, n. 118, p. 1 - 24, 2007.

LORENZI, H. Árvores brasileiras: manual de identificação e cultivo de plantas arbóreas nativas do Brasil. Nova Odessa: Editora Plantarum, 1992. 352 p.

MACHADO, V. J.; SOUZA, C. H. E.; ANDRADE, B. B.; LANA, R. M. Q.; KORNDORFER, G. H. Curvas de disponibilidade de fósforo em solos com diferentes texturas após aplicação de doses crescentes de fosfato monoamônico. Bioscience Journal, Uberlândia, v. 27, n. 1, p. 70 - 76, 2011. 
MALAVOLTA, E.; VITTI, G. C.; OLIVEIRA, S. A. Avaliação do estado nutricional das plantas: princípios e aplicações. 2. ed. Piracicaba: POTAFOS, 1997. 319 p.

MELO, L. C. A.; SILVA, C. A. Influência de métodos de digestão e massa de amostra na recuperação de nutrientes em resíduos orgânicos. Química Nova, São Paulo, v. 31, n. 3, p. 556 - 561, 2008.

MORRISON, D. F. Multivariate statical methods. New York: McGraw-Hill Book CO., 1967. 415 p.

NICOLOSO, F. T.; FOGAÇA, M. A. F.; ZANCHETI, F.; FORTUNATO, R. P.; MISSIO, E. L. Exigências nutricionais da grápia em Argissolo Vermelho distrófico arênico: efeito da adubação NPK no teor de nutrientes nos tecidos. Revista Ciência Agronômica, Fortaleza, v. 40, n. 3, p. 417 - 426, 2009.

OLIVEIRA, R. E. Aspectos da dinâmica de um fragmento florestal em Piracicaba, SP: silvigênese e ciclagem de nutrientes. 79 f. Dissertação (Mestrado em Ciências Florestais) - Escola Superior de Agricultura Luiz de Queiroz, Piracicaba, 1997.

OSÓRIO FILHO, B. D. Dinâmica de enxofre no sistema solo e resposta das culturas à adubação sulfatada. 76 f. Dissertação (Mestrado em Ciência do Solo) - Universidade Federal de Santa Maria, Santa Maria, 2006.

PANDOLFO, C. M.; FLOSS, P. A.; CROCE, D. M.; DITTRICH, R. C. Resposta da erva-mate (Ilex paraguariensis St.-Hil.) à adubação mineral e orgânica em um Latossolo Vermelho aluminoférrico. Ciência Florestal, Santa Maria, v. 13, n. 2, p. 37 - 45, 2003.

REISSMANN, C. B.; ROCHA, H.; KOCHLER, C. W.; CALDAS, R. L. S.; HILDEBRAND, E. E. Bioelementos em folhas e hastes de erva-mate (Ilex paraguariensis St.-Hil.) sobre cambissolos na região de Mandirituba, PR. Floresta, Curitiba, v. 16, n. 2, p. 49 - 54, 1983.

RHEINHEIMER, D. S.; ANGHINONI, I.; CONTE, E. Fósforo da biomassa microbiana em solos sob diferentes sistemas de manejo. Revista Brasileira de Ciência do Solo, Viçosa, v. 24, n. 3, p. 589 - 597 , 2000.

RIZZINI, C. T. Árvores e madeiras úteis do Brasil: manual de dendrologia brasileira. São Paulo: Edgard Blucher, 1979. 296 p.

SCOLFORO, J. R. S.; OLIVEIRA, A. D.; DAVIDE, A. C. Manejo sustentado das candeias Eremanthus erytropappus (DC.) McLeish e Eremathus incanus (Less.). Disponível em: <www.nucleo estudo.ufla.br/nemaf/candeia>. Acesso em: 15/10/2011.

SILVA, A. C.; ROSADO, S. C. S.; VIEIRA, C. T.; CARVALHO, D. Variação genética entre e dentro de populações de candeia (Eremanthus erythropappus (DC) MacLeish). Ciência Florestal, Santa Maria, v. 17, n. 3, p. $271-277,2007$.

SILVA, F. C. Manual de análises químicas de solos, plantas e fertilizantes. 2. ed. Brasília: Embrapa Informações Tecnológicas, 2009. 627 p.

SOUZA, C. A. M.; OLIVEIRA, R. B.; FILHO, S. M.; LIMA, J. S. S. Crescimento em campo de espécies florestais em diferentes condições de adubação. Ciência Florestal, Santa Maria, v. 16, n. 3, p. 243 - 249, 2006.

VARANINE, Z.; PINTON, R.; BIASE, M. G.; ASTOLFI, S.; MAGGIONI, A. Low molecular weight humic substances stimulate $\mathrm{H}^{+}$-ATPase activity of plasma membrane vesicles isolated from oat (Avena sativa L.) roots. Plant and Soil, Netherlands, v. 153, n. 3, p. 61 - 69, 1993.

VAZ, L. M. S.; GONÇALVES, J. L. M. Uso de biossólidos em povoamento de eucalipto: efeito em atributos químicos do solo, no crescimento e na absorção de nutrientes. Revista Brasileira de Ciência do Solo, Viçosa, v. 26, n. 3, p. 747 - 758, 2002.

VENTURIN, N.; SOUZA, P. A.; MACEDO, R. L. G.; NOGUEIRA, F. D. Adubação mineral da candeia (Eremanthus erythropappus (DC.) McLeish). Floresta, Curitiba, v. 35, n. 2. p. 211 - 219, 2005.

VENTURIN, N.; SOUZA, P. A.; VENTURIN, R. P. Avaliação nutricional da candiúva (Trema micrantha L. Blumes) em casa de vegetação. Floresta, Curitiba, v. 29, n. 1-2, p. 15 - 26, 2000. 
WALlAU, R. L. R.; BORGES, A. R.; ALMEIDA, D. R.; CAMARGOS, S. L. Sintomas de deficiências nutricionais em mudas de mogno cultivadas em solução nutritiva. Cerne, Lavras, v. 14, n. 4, p. 304 - 310, 2008.

WILLIAMS, E. D. Changes during three years in the size and composition of seed bank beneath a longterm pasture as influenced by defoliation and fertilizer regime. Journal of Applied Ecology, London, v. 21, n. 2, p. 603 - 615, 1984.

WILLIAMSON, J. C.; ROWE, E. C.; HILL, P. W.; NASON, M. A.; JONES, D. L.; HEALEY, J. R. Alleviation of both water and nutrient limitations is necessary to accelerate ecological restoration of waste rock tips. Restoration Ecology, Malden, v. 19, n. 2, p. 194 - 204, 2011. 